\title{
Il peso secco nel bambino in emodialisi
}

\author{
Silvia Consolo, Fabio Paglialonga, Alberto Edefonti \\ UOC Nefrologia e Dialisi Pediatrica, Fondazione IRCCS Ca' Granda Ospedale Maggiore Policlinico, Milano
}

\begin{abstract}
Dry weight in children on hemodialysis
An accurate assessment of dry-weight (DW) is critical in children on chronic hemodialysis (HD) to avoid the risk of both volume overload and volume depletion. It requires a comprehensive evaluation, which includes clinical history, physical examination and diagnostic tools, such as bioimpedance analysis and non invasive volume monitoring. An optimal sodium balance, an adequate interdialytic weight gain and intensified HD schedules are the key points to reach DW. This review focuses on the risk of volume overload and depletion, on the methods of DW assessment and on the best strategies to reach DW in children on HD.
\end{abstract}

Keywords: Pediatric hemodialysis, Dry weight, Volume monitoring, Bioimpedance analysis

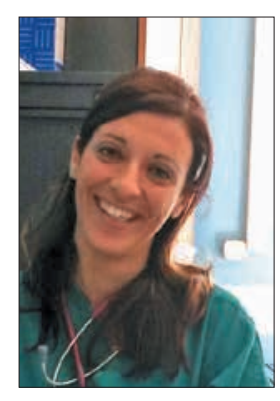

Silvia Consolo

\section{Introduzione}

Nonostante i progressi nella gestione del paziente pediatrico con insufficienza renale terminale, le problematiche cardiovascolari rimangono una causa comune di morbidità e mortalità. L'identificazione corretta del peso secco (Dry Weight, DW), vale a dire del peso ottimale al termine della seduta di emodialisi (ED), è un requisito indispensabile per ridurre il rischio di sovraccarico o di deplezione eccessiva di volume. Tuttavia, la stima del DW è complicata dalla mancanza di un gold-standard per la valutazione della composizione corporea e dalle modificazioni del peso in relazione alla crescita. Infine, l'emodialisi trisettimanale rende spesso difficile il raggiungimento del DW, esponendo il paziente al rischio di sovraccarico di volume oppure alla necessità di ultrafiltrazione aggressiva con morbidità intradialitica.

Accepted: August 10, 2015

Published online: October 14, 2015

Indirizzo per la corrispondenza:

Silvia Consolo

UOC Nefrologia e Dialisi Pediatrica

Fondazione IRCCS Ca' Granda

Ospedale Maggiore Policlinico

Via Commenda 9

20122 Milano

silvia.consolo@alice.it
Scopo della presente revisione è di discutere in merito alla definizione di DW, ai metodi disponibili per identificarlo e alle strategie ottimali per raggiungerlo nel bambino in ED.

\section{Definizione di peso secco}

II DW è definito come "il peso al termine della dialisi che permette il raggiungimento di normali valori di pressione arteriosa all'inizio e al termine della dialisi senza farmaci antipertensivi, senza segni clinici di iper- e ipoidratazione e nonostante l'aumento di peso interdialitico" o come "il più basso peso post-dialitico tollerato al quale siano minimizzati i segni e i sintomi di ipervolemia o ipovolemia" $(1,2)$. II concetto sottostante è che il DW è il peso post-ED che consente di mantenere il miglior equilibrio tra sovraccarico e deplezione di volume.

\section{Sovraccarico di volume}

Il sovraccarico di volume del paziente in emodialisi è composto da due componenti: l'aumento di peso interdialitico (Interdialytic Weight Gain, IDWG) e un eventuale eccesso di volume cronico post-dialitico. La relazione tra sovraccarico di volume e complicanze cardiovascolari è ben dimostrata. In uno studio su 71 pazienti pediatrici in emodialisi, 42 dei quali ipertesi, l'eccesso di peso post-ED rispetto al DW era significativamente più alto nei bambini ipertesi che in quelli normotesi ( $1.85 \%$ vs $0.46 \%$, p 0.008) (3). Recentemente, è stata dimostrata una correlazione significativa tra IDWG e indice di massa del ventricolo sinistro: nessuno dei bambini con un IDWG inferiore al $4 \%$ del peso corporeo presentava ipertrofia ventricolare sinistra, contro l'83.3\% di quelli con IDWG superiore (4). 
Tra le complicanze cardiovascolari, l'ipertrofia concentrica è la conseguenza di un prolungato sovraccarico di pressione, che porta a un ispessimento della tonaca muscolare e a una contemporanea riduzione del diametro della cavità. Entrambi determinano, quindi, una conseguente diminuzione finale della capacità di distensione ventricolare. Invece, l'ipertrofia eccentrica è riconducibile a un prolungato sovraccarico di volume, che conduce a un aumento dello spessore parietale, associato, però, a un contemporaneo incremento del diametro della sua cavità, pertanto il rapporto spessore/raggio si mantiene entro il range di normalità. In analogia, anche un ampio flusso proveniente da una fistola arterovenosa confezionata come accesso vascolare può condurre a un rimodellamento cardiaco e, quindi, a un quadro di ipertrofia ventricolare eccentrica o asimmetrica.

Inoltre, il sovraccarico di volume è associato ad astenia, riduzione della capacità di esercizio e inappetenza. La riduzione dell'apporto alimentare può, a sua volta, condurre a una perdita di massa muscolare e, qualora non venga abbassato il peso post-ED, a un ulteriore peggioramento dell'iperidratazione. La riduzione della frequenza scolastica è comune nel bambino in eccesso di volume, per motivi sia organici che psicologici, così come è comune un peggioramento della qualità di vita.

Infine, le complicanze tardive associate a un controllo del peso non adeguato sono prevalentemente di natura cardiovascolare. Infatti, l'ipertrofia ventricolare sinistra è molto più frequente nei pazienti che hanno intrapreso la dialisi dall'infanzia ed espone a un aumentato rischio di conseguenze potenzialmente letali, come infarto miocardico o aritmie. Queste ultime rappresentano la principale causa di decesso in questi pazienti.

\section{Deplezione di volume}

Un'eccessiva rimozione di acqua e sodio può portare a sintomi intradialitici da ipoperfusione, tra cui crampi, nausea, vomito e dolori addominali, che possono ridurre l'accettazione della metodica. In altre occasioni, l'ipovolemia può manifestarsi con ipotensione arteriosa, talora severa, con il rischio di danno d'organo, per ischemia cerebrale, gastrointestinale o cardiaca (5).

Negli ultimi anni è stata posta attenzione al "myocardial stunning", ovvero al fenomeno di ischemia miocardica acuta associata a disfunzione regionale del ventricolo sinistro causata dall'ipoperfusione. Uno studio su 12 bambini in ED sottoposti a ecocardiografie seriate ha dimostrato la presenza di stunning di vario grado in $11 / 12$ pazienti (6).

Eventi ipotensivi possono, inoltre, portare a trombosi a carico della fistola arterovenosa o della protesi vascolare e alla perdita della funzione renale residua. Va, infine, considerato come l'eccessiva deplezione di volume si associ a un aumento della sete, con peggioramento della qualità di vita, e a un aumento dell'introduzione interdialitica di liquidi.

\section{Valutazione del peso secco}

La valutazione del DW nel bambino non può prescindere dall'anamnesi raccolta all'inizio di ogni dialisi, volta a indagare l'apporto alimentare ed eventuali patologie intercorrenti. Va, inoltre, tenuta in considerazione l'eventuale sintomatologia intradialitica precedente.

La pressione arteriosa è di estrema importanza, ma la sua misurazione estemporanea all'inizio della dialisi è un marker non adeguato di ipertensione, se interpretato come singola misurazione. Uno studio su 17 bambini in ED ha dimostrato come una diagnosi di ipertensione fosse possibile nel $24 \%$ dei pazienti secondo la misurazione pressoria estemporanea e nel $59 \%$ dei pazienti secondo il monitoraggio delle 44 ore. Inoltre, l'indice di massa del ventricolo sinistro era significativamente correlato con i valori del monitoraggio continuo, ma non si correlava con i valori estemporanei (7).

Anche l'esame obiettivo è poco sensibile per la determinazione del peso secco. Tra i segni clinici peculiari di deplezione di volume va segnalata la modifica del timbro della voce (8).

In sintesi, anamnesi ed esame obiettivo sono indispensabili ma non sufficienti. È necessario, pertanto, fare ricorso a tecniche diagnostiche, come l'analisi di bioimpedenza (BIA), il diametro della vena cava inferiore (IVCD), i peptidi natriuretici e il monitoraggio non invasivo della volemia (BVM). Tra queste, la BIA punta a quantificare l'acqua totale corporea, mentre tutte le altre tecniche si focalizzano sul compartimento intravascolare.

\section{Analisi di bioimpedenza (BIA)}

La BIA è una metodica di valutazione della composizione corporea basata sull'utilizzo di una corrente alternata, che, nel suo passaggio nei tessuti, incontra fluidi, che offrono una resistenza, e membrane cellulari, che si comportano come elementi capacitivi. Prevede il posizionamento di 4 elettrodi, 2 dei quali alla mano e 2 al piede omolaterale. L'approccio in monofrequenza sfrutta una corrente di $50 \mathrm{kHz}$ e permette di ottenere un valore di impedenza, a sua volta scomposto nelle due componenti di Resistenza e Reattanza. Sono stati tentati vari approcci per l'interpretazione di questi indici anche nel bambino, senza evidenti dimostrazioni di efficacia. Recentemente, è stato proposto un approccio per la stima del DW nel bambino a partire dai semplici valori bioimpedenziometrici misurati a fine trattamento, basato sulla misurazione longitudinale di tali indici, in modo che ogni soggetto funga da controllo di se stesso (9). Per ogni paziente viene identificato un range di valori di Resistenza e Reattanza post-dialitici ideali: valori superiori al range suggeriscono deplezione di volume, mentre indici troppo bassi indicano un persistente eccesso, con la necessità di ridurre il DW. Tale approccio ha consentito, in una casistica pediatrica, di ridurre l'indice di massa del ventricolo sinistro, la prevalenza di ipertrofia ventricolare sinistra e l'incidenza di edema polmonare acuto (9). 
Negli ultimi anni la bioimpedenza in multifrequenza (spettroscopia) ha ricevuto maggiore attenzione, poiché, tramite l'utilizzo di una corrente alternata a frequenze multiple, consente di dare un'idea della distribuzione dell'acqua corporea. Non esistono vere dimostrazioni di accuratezza di tale metodica in ambito pediatrico. In una delle poche applicazioni in pazienti pediatrici è stata studiata la relazione tra valori di pressione arteriosa e stato di idratazione, valutato in predialisi tramite BIA in multifrequenza, in 23 bambini in ED (10). All'inizio della seduta era riscontrabile uno stato di ipoidratazione nel 5.5\% dei casi, nel $62.4 \%$ dei casi un quadro di normoidratazione, mentre un'iperidratazione moderata o severa era riscontrata nel $21 \%$ e nell' $11.2 \%$ delle sedute, rispettivamente. Non vi era alcuna correlazione tra pressione arteriosa e stato di idratazione, suggerendo che la stima del peso secco basata solo sui valori della pressione arteriosa può essere sbagliata (10).

\section{Diametro della vena cava inferiore}

La misurazione ecografica dell'IVCD è stata proposta come stima dello stato di idratazione anche nel bambino. Un primo studio ha dimostrato un maggior IVCD e una ridotta collassabilità della vena cava inferiore in 12 pazienti pediatrici in emodialisi rispetto a 12 controlli (11). In 15 bambini in ED sono state, poi, dimostrate una significativa riduzione dell'IVCD durante il trattamento e una correlazione significativa tra le variazioni dell'IVCD e le variazioni di peso durante la seduta; l'indice di collassabilità (la modifica percentuale di diametro della vena cava inferiore in espirazione vs inspirazione) aumentava significativamente durante la dialisi (12). Sebbene queste evidenze suggeriscano una potenziale utilità, la mancanza di valori di riferimento e le notevoli variazioni interindividuali rappresentano precisi limiti. Oltretutto, va sottolineato come I'IVCD rifletta unicamente il volume intravascolare: se effettuato al termine del trattamento, prima che si sia verificato il refill dei fluidi nel distretto intravascolare, non può essere considerato come stima dell'acqua totale corporea.

\section{Peptidi natriuretici}

Tra i peptidi natriuretici, il Brain Natriuretic Peptide (BNP) e la sua frazione N-terminale (NT-BNP) sono quelli che hanno suscitato più interesse per la stima del DW. Diversi studi nell'adulto hanno analizzato il ruolo di tali indici come marker indipendenti di volume, con risultati contraddittori $(13,14)$ : si tratta soprattutto di studi piccoli, trasversali, limitati da valutazioni dello stato di idratazione condotte con metodiche non sempre affidabili. Nel DRIP Trial 150, pazienti adulti in ED sono stati randomizzati a ultrafiltrazione intensiva o a gestione standard: non vi era alcuna correlazione tra le modifiche del peso e le variazioni di BNP, a suggerirne una scarsa attendibilità come indice di volume nel dializzato (14). Considerata anche l'assenza di dati pediatrici, non vi è, al momento, alcuna evidenza per suggerirne l'utilizzo per la stima del DW nel bambino.

\section{Monitoraggio non invasivo della volemia (BVM)}

Tale metodica consiste nella valutazione delle variazioni di volemia durante il trattamento, basata sulla misurazione continua dell'ematocrito. Il razionale è che le variazioni di volemia nel dializzato dipendono dalla velocità di ultrafiltrazione e dalla velocità di refill, a loro volta funzioni della pressione idrostatica (ovvero del sovraccarico di volume) e della pressione oncotica plasmatica. Un'eccessiva caduta della volemia può dipendere da un'eccessiva velocità di ultrafiltrazione e da una bassa pressione oncotica (ipoalbuminemia) o deplezione di volume. II BVM fornisce tre informazioni utili sia per la prevenzione dell'ipotensione intradialitica sia per l'identificazione di pazienti con sovraccarico di volume.

II tipo di curva del BVM rappresenta il primo elemento da considerare (Fig. 1). In uno studio su 2240 analisi di BVM in pazienti pediatrici, il 91\% delle sedute, tutte asintomatiche, seguiva lo stesso pattern di variazione della volemia, con una riduzione iniziale seguita da una stabilità (15). In una minoranza di casi, era presente un andamento differente, con scarsa caduta della volemia, indicativa di sovraccarico di volume, oppure con caduta continua e rapida, suggestiva del rischio di ipovolemia e di una sintomatologia intradialitica (Fig. 1).

Il secondo elemento da considerare è il valore percentuale di riduzione della volemia. Studi pediatrici indicano come adeguata una caduta della volemia inferiore all' $8 \%$ orario nei primi 90 minuti e una caduta inferiore al $4 \%$ orario nel resto della seduta (16).

II terzo elemento è la capacità di refill, che può essere stimata sospendendo l'ultrafiltrazione alla fine del trattamento: in presenza di un eccesso di liquidi nell'interstizio, entro 10 minuti dalla sospensione dell'ultrafiltrazione, si verifica un significativo refill che determina un rapido aumento della volemia, il che non si verifica, invece, in assenza di un eccesso di volume (5) (Fig. 2).

Negli ultimi anni, sono state pubblicate diverse esperienze in emodialisi pediatrica. In uno studio prospettico su 20 bambini in ED, l'applicazione di un protocollo di ultrafiltrazione basato sul BVM ha consentito di ridurre, nell'arco di sei mesi, i valori della pressione arteriosa, il numero di farmaci antipertensivi e l'incidenza di complicanze intradialitiche (17). II BVM può essere utile anche nel paziente acuto in ED, come dimostrato da uno studio retrospettivo su 23 bambini con insufficienza renale acuta, in cui è stato confrontato l'andamento di 70 sedute assistite da BVM rispetto a 64 in cui il BVM non era stato utilizzato: in assenza di differenze nelle incidenza di episodi ipotensivi intradialitici tra i due gruppi, l'entità di ultrafiltrazione era più alta nelle sedute BVM-assistite rispetto a quelle di controllo. Nelle sedute senza ipotensione la caduta della volemia era sempre inferiore al $13 \%$ (18).

In conclusione, I'utilizzo del BVM appare un metodo affidabile per l'identificazione di pazienti a rischio di complicanze intradialitiche e per l'identificazione del DW nel bambino in ED. 


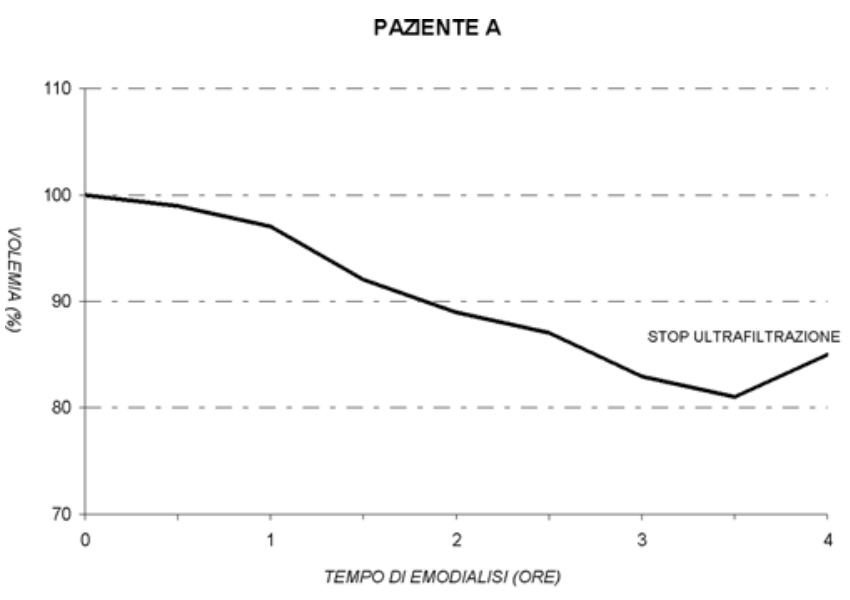

PAZENTE B

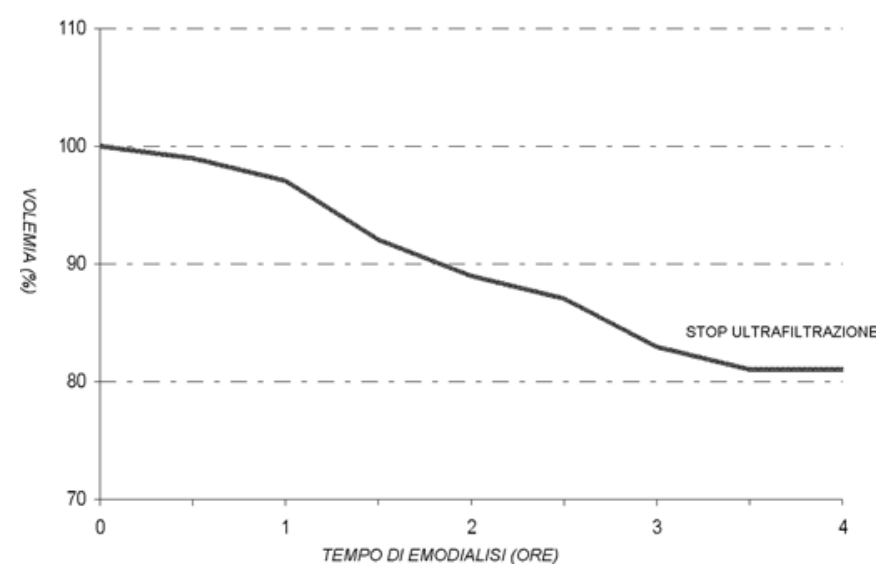

Fig. 1 - Rappresentazione dei 3 possibili tipi di curva nel monitoraggio non invasivo della volemia con BVM: curva 1, normale, curva 2, a rischio di sovraccarico di volume, curva 3 , a rischio di ipovolemia.

\section{Strategie per il raggiungimento del peso secco nel bambino}

Gli schemi di dialisi più comuni (3.5-4 ore per 3 volte a settimana) non consentono frequentemente di raggiungere il peso ottimale nei bambini con limitata capacità di ultrafiltrazione ed elevato apporto interdialitico di liquidi. Alcune strategie possono essere attuate per ridurre l'IDWG e ottimizzare l'ultrafiltrazione durante il trattamento. La risposta migliore al problema del controllo del volume nel paziente in ED rimane, comunque, la dialisi intensificata.

\section{Riduzione dell'aumento di peso interdialitico}

La principale strategia per ridurre I'IDWG consiste nel ridurre l'apporto di sodio interdialitico (19). È, infatti, ben dimostrata la correlazione diretta tra apporto di sodio interdialitico e apporto di liquidi. La restrizione alimentare di sodio può, effettivamente, portare a una riduzione di IDWG, pressione arteriosa e massa ventricolare sinistra (20).

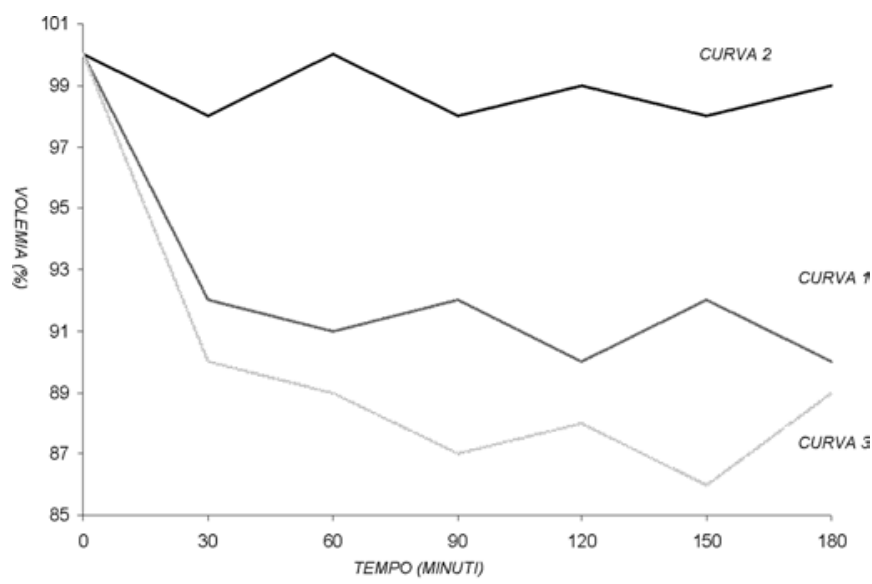

CURVA 1: NORMALE

CURVA 2: RISCHIO DI SOVRACCARICO DI VOLUME

CURVA 3: RISCHIO DI IPOVOLEMIA

Fig. 2 - Stima della capacità di refill a fine dialisi tramite monitoraggio della volemia con BVM: paziente $A$, non a peso secco, paziente $B$, arrivato a peso secco.

Data la frequente non aderenza alla dieta del paziente pediatrico, molto interesse si è concentrato sulla possibilità di ottimizzare il bilancio del sodio agendo sulla concentrazione di sodio del dialisato $(\mathrm{dNa})$.

La rimozione intradialitica di sodio consta di una componente convettiva, associata all'ultrafiltrazione, e di una componente diffusiva, funzione del gradiente di concentrazione tra plasma e dialisato. Con gli schemi di dialisi più comuni e valori di dNa tra 138 e $140 \mathrm{mEq} / \mathrm{L}$, circa l' $80 \%$ della rimozione di sodio è affidata alla convezione. Concentrazioni più elevate di $\mathrm{dNa}$ o il profilo del sodio vengono utilizzati per ridurre la sintomatologia intradialitica, ma sono associati a un bilancio del sodio intradialitico più positivo, con aumento della sete e della pressione arteriosa e sovraccarico di volume. Al contrario, valori di dNa più bassi portano a un bilancio intradialitico di sodio più negativo, ma sono accompagnati da un aumento della sintomatologia intradialitica.

L'individualizzazione di dNa costituisce la strategia migliore (21), complicata, però, dall'estrema variabilità della natriemia del paziente. In uno studio su 23 bambini in ED, la media di 905 determinazioni di Na plasmatico predialisi era pari a $138 \pm 1.4$ $\mathrm{mmol} / \mathrm{L} / \mathrm{L}$, significativamente più bassa rispetto al $\mathrm{dNa}$ prescritto ( $140 \pm 2.1 \mathrm{mmol} / \mathrm{L}, \mathrm{p} 0.002)$. La natriemia preED variava da 135.6 \pm 3.12 a $140.2 \pm 1.6 \mathrm{mmol} / \mathrm{L}$, con un'estrema variabilità intra- e interindividuale (10). In conclusione, una prescrizione del dNa individualizzata può costituire una risorsa terapeutica per ridurre sete, IDWG e sovraccarico di volume, ma è gravata dal rischio di riduzione della tolleranza dialitica.

\section{Strategie per ottimizzare l'ultrafiltrazione}

Pochi studi hanno valutato nel bambino le strategie ottimali per ridurre gli episodi ipotensivi o la sintomatologia intradialitica in caso di necessità di un'ultrafiltrazione elevata. 
Uno studio su 506 sedute in 10 pazienti pediatrici ha confrontato l'effetto di profili del sodio, profili di ultrafiltrazione e infusione intradialitica di mannitolo. L'ultrafiltrazione media era costituita dal 5.4\% del peso, con un massimo pari al 9.7\%. Un profilo di dNa a gradini (da 148 a $138 \mathrm{mmol} / \mathrm{L}$ ) riduceva il rischio di sintomatologia intradialitica e di ipotensione rispetto a un profilo lineare. L'infusione di mannitolo si associava a una riduzione del $64 \%$ del rischio di sintomatologia intradialitica, senza effetti sul rischio di ipotensione. Tutti i profili di ultrafiltrazione erano associati a un aumento del rischio di sintomatologia intradialitica, probabilmente per gli elevati livelli di ultrafiltrazione. Nonostante tutte le strategie implementate, i livelli di ultrafiltrazione raggiunti erano inferiori all'IDWG nel 66\% dei casi (22). In conclusione, con gli schemi di dialisi intermittente più diffusi, le strategie disponibili non consentono, in un'elevata percentuale di trattamenti, di portare il bambino al DW desiderato.

\section{Emodialisi intensificata}

Anche nel bambino gli schemi di dialisi intensificata hanno rivestito grande interesse negli ultimi anni. Diversi studi hanno confermato che regimi di emodialisi o emodiafiltrazione quotidiana si associano a un miglior controllo pressorio, alla riduzione del fabbisogno di farmaci antipertensivi, alla possibilità di liberalizzare la dieta con un incremento degli apporti nutrizionali, al miglioramento della fosforemia e a un significativo recupero della velocità di crescita $(23,24)$. A oggi, la dialisi quotidiana, ospedaliera o domiciliare costituisce la risposta migliore al problema del controllo dei volumi.

\section{Conclusioni}

Il problema dell'identificazione e della gestione del peso secco riveste grande interesse nel bambino, ai fini della riduzione del rischio sia di deplezione del volume, causa di morbidità intradialitica, sia di eccesso di volume, responsabile di ipertensione arteriosa e complicanze cardiovascolari. L'identificazione del DW ottimale è gravata dalla scarsa sensibilità dell'esame obiettivo e dalla mancanza di una metodica gold-standard per la stima della composizione corporea. L'analisi di bioimpedenza e, soprattutto, il monitoraggio non invasivo della volemia in corso di dialisi sono strumenti utili. II raggiungimento del DW non può prescindere da un'ottimizzazione del bilancio del sodio, raggiunta sia attraverso una riduzione dell'apporto alimentare sia attraverso un'individualizzazione della concentrazione di sodio del dialisato. Schemi di dialisi intensificata, che utilizzano, in particolare, l'emodiafiltrazione quotidiana, rappresentano la migliore strategia disponibile per risolvere il problema della gestione dei volumi nel bambino in emodialisi.

\section{Disclosures}

Financial support: No financial support was received for this submission. Conflict of interest: The authors have no conflict of interest.

\section{Bibliografia}

1. Hecking $M$, Karaboyas A, Antlanger M, et al. Significance of interdialytic weight gain versus chronic volume overload: consensus opinion. Am J Nephrol 2013;38:78-90.

2. Charra B, Laurent G, Chazot C, et al. Clinical assessment of dry weight. Nephrol Dial Transplant 1996;11(Suppl. 2):16-9.

3. Van DeVoorde RG, Barletta GM, Chand DH, et al. Blood pressure control in pediatric hemodialysis: the Midwest Pediatric Nephrology Consortium Study. Pediatr Nephrol 2007;22:547-53.

4. Paglialonga F, Consolo S, Galli MA, Testa S, Edefonti A. Interdialytic weight gain in oligoanuric children and adolescents on chronic hemodialysis. Pediatr Nephrol 2015;30:999-1005.

5. Hayes W, Hothi DK. Intradialytic hypotension. Pediatr Nephrol 2011;26:867-79.

6. Hothi DK, Rees L, Marek J, Burton J, McIntyre CW. Pediatric myocardial stunning underscores the cardiac toxicity of conventional hemodialysis treatments. Clin J Am Soc Nephrol 2009;4:790-7.

7. Katsoufis CP, Seeherunvomng W, Sasaki N, et al. Forty-four-hour interdialytic ambulatory blood pressure monitoring and cardiovascular risk in pediatric hemodialysis patients. Clin Kidney J 2014;7:33-9.

8. Unver S, Hardal U, Esertas K, Sezen A, Celikbilek F, Altundag A Objective analysis of voice changes in a hemodialysis session and its correlation with ultrafiltration. Ren Fail 2014;2:1-5.

9. Paglialonga F, Ardissino G, Galli MA, Scarfia RV, Testa S, Edefonti A. Bioimpedance analysis and cardiovascular status in pediatric patients on chronic hemodialysis. Hemodial Int 2012;16(Suppl. 1):S20-5.

10. Zaloszyc A, Schaefer B, Schaefer F, et al. Hydration measurement by bioimpedance spectroscopy and blood pressure management in children on hemodialysis. Pediatr Nephrol 2013;28:2169-77.

11. Sonmez F, Mir S, Ozyiirek AR, Cura A. The adjustment of post-dialysis dry weight based on non-invasive measurements in children. Nephrol Dial Transplant 1996;11:1564-7.

12. Krause I, Birk E, Davidovits M, et al. Inferior vena cava diameter for estimation of fluid status in children on haemodialysis. Nephrol Dial Transplant 2001;16:1203-6.

13. Booth J, Prinney J, Davenport A. N-terminal proBNP-marker of cardiac dysfunction, fluid overload, or malnutrition in hemodialysis patients? Clin J Am Soc Nephrol 2010;5:1036-40.

14. Agarwal R. B-type natriuretic peptide is not a volume marker among patients on hemodialysis. Nephrol Dial Transplant 2013;28:3082-9.

15. Dheu C, Terzic J, Menouer S, Fischbach M. Importance of the curve shape for interpretation of blood volume monitor changes during haemodiafiltration. Pediatr Nephrol 2009;24: 1419-23.

16. Jain SR, Smith L, Brewer ED, Goldstein SL. Non-invasive intravascular monitoring in the pediatric hemodialysis population. Pediatr Nephrol 2001;16:15-8.

17. Patel HP, Goldstein SL, Mahan JD, et al. A standard, noninvasive monitoring of hematocrit algorithm improves blood pressure control in pediatric hemodialysis patients. Clin J Am Soc Nephrol 2007;2:252-7.

18. Merouani A, Kechaou W, Litalien C, Ducruet T, Jouvet P. Impact of blood volume monitoring on fluid removal during intermittent hemodialysis of critically ill children with acute kidney injury. Nephrol Dial Transplant 2011;26:3315-9.

19. Tomson CR. Advising dialysis patients to restrict fluid intake without restricting sodium intake is not based on evidence and is a waste of time. Nephrol Dial Transplant 2001;16:1538-42.

20. Kayikcioglu M, Tumuklu M, Ozkahya $M$, et al. The benefit of salt restriction in the treatment of end-stage renal disease disease by haemodialysis. Nephrol Dial Transplant 2009;24: 956-62.

21. Santos SF, Peixoto AJ. Revisiting the dialysate sodium prescription as a tool for better blood pressure and interdialytic weight gain management in hemodialysis patients. Clin J Am Soc Nephrol 2008;3:522-30.

22. Hothi DK, Harvey E, Goia CM, Geary DF. Evaluating methods for improving ultrafiltration in pediatric hemodialysis. Pediatr Nephrol 2008;23:631-8.

23. Fischbach M, Terzic J, Laugel V, et al. Daily on-line haemodiafiltration: a pilot trial in children. Nephrol Dial Transplant 2004;19:2360-7.

24. Goldstein SL, Silverstein DM, Leung JC, et al. Frequent hemodialysis with NxStage system in pediatric patients receiving maintenance hemodialysis. Pediatr Nephrol 2008;23:129-35. 